\title{
High-resolution capillary zone electrophoresis for transferrin glycoform analysis associated with congenital disorders of glycosylation
}

\author{
Micha Tobler $^{1, \S}$, Jitka Caslavska ${ }^{2, \S}$, Patricie Burda ${ }^{1}$, Wolfgang Thormann ${ }^{2}$ \\ 1) Division of Metabolism, University Children's Hospital Zürich, Zürich, Switzerland. \\ 2) Clinical Pharmacology Laboratory, Institute for Infectious Diseases, University of Bern, \\ Bern, Switzerland.
}

Short title: CE for transferrin analysis in congenital disorders of glycosylation

Abbreviations: CDG, congenital disorder of glycosylation; CDT, carbohydrate-deficient transferrin; COG, conserved oligomeric Golgi; GIEF, gel isoelectric focusing; PGM1, phosphoglucomutase 1; PMM2, phosphomannomutase 2; Tf, transferrin

Keywords: capillary electrophoresis, congenital disorder of glycosylation, desialylated transferrin, transferrin glycoforms

* Author, to whom correspondence should be addressed:

Professor Dr. Wolfgang Thormann

Clinical Pharmacology Laboratory, Murtenstrasse 35, CH-3008 Bern, Switzerland phone: +4131632 3288; fax: +41316324997

email: wolfgang.thormann@ifik.unibe.ch

$\S$ These authors contributed equally to this work.

Received: 01 23, 2018; Revised: 04 18, 2018; Accepted: 04 19, 2018

This article has been accepted for publication and undergone full peer review but has not been through the copyediting, typesetting, pagination and proofreading process, which may lead to differences between this version and the Version of Record. Please cite this article as doi: $10.1002 /$ jssc. 201800082 .

This article is protected by copyright. All rights reserved. 


\section{Abstract}

High-resolution capillary zone electrophoresis is used to assess the transferrin profile in serum of patients with eight different congenital disorders of glycosylation that represent type I, type II and mixed type I/II disorders. Capillary zone electrophoresis data are compared to patterns obtained by gel isoelectric focusing. The high-resolution capillary zone electrophoresis method is shown to represent an effective tool to assess the diversity of transferrin patterns. Hypoglycosylated disialo-, monosialo- and asialo-transferrin in type I cases can be distinguished from the corresponding underdesialylated transferrin glycoforms present in type II disorders. The latter can be separated from and are detected ahead of their corresponding hypoglycosylated forms of type I patients. Both types of glycoforms are detected in sera of mixed type I/II patients. The assay has the potential to be used as screening method for congenital disorders of glycosylation. It can be run with a few $\mu \mathrm{L}$ of serum when microvials are used.

\section{INTRODUCTION}

Transferrin (Tf) is an iron transporting glycoprotein with two iron binding sites and two Nlinked carbohydrate chains which are composed of a bi- to tetraantennary carbohydrate chain with terminal sialic acid residues. Due to different numbers of end standing sialic acid residues (zero up to eight), human serum comprises Tf isoforms which differ in their pl values (between 5.9 and about 5.0 after complete iron saturation) and electrophoretic mobilities [1,2]. Monitoring of Tf isoforms is important in clinical and forensic analysis for the determination of carbohydrate-deficient transferrin (CDT) which is a marker for 
excessive alcohol intake over an extensive period of time, the elucidation of genetic variants, the monitoring of asialo-Tf (referred to as beta-2-Tf ) in nasal and other fluids which is a marker for cerebrospinal fluid leakage, and the assessment of a congenital disorder of glycosylation (CDG). CDGs are a group of rare recessively inherited diseases with severe neurological and/or systemic manifestations from early childhood [3-6]. CDGs are divided into two main groups: type I CDGs comprise defects in the assembly of the dolichol lipid-linked oligosaccharide chain and its transfer to the nascent protein and are characterized by unoccupied glycosylation sites on proteins, thus lacking complete Nglycans. Type II CDGs refer to defects in the trimming and processing of the protein-bound glycans either late in the endoplasmic reticulum or the Golgi compartments resulting in immature, truncated glycans. Gel isoelectric focusing (GIEF) [1,2,7,8], gel electrophoresis [9], HPLC [10], CZE [11-17] and MS [18-22] are employed to measure Tf isoform distributions in human serum.

CZE assays allow separation of Tf isoforms on the basis of differences in electrophoretic mobilities, i.e. charge/mass with the charge state being determined by the number of terminal sialic acid residues. They are attractive for CDG screening as they can be automated and require small amounts of serum only. The latter aspect, which is an important feature as only tiny amounts of serum can be drawn from newborns and infants, is also given in GIEF. Tf isoform analysis by CZE is performed with automated multicapillary platforms (CAPILLARYS of Sebia, Evry, France) or with the CDT reagent kit CEofix (Analis, Namur, Belgium) on single capillary instruments of different manufacturers $[23,24]$. The latter 
system comprises polymeric polyions that form a dynamic double coating, an approach that leads to highly reproducible data [16] and was optimized for high-resolution analyses in our laboratory [17] which is advantageous for analysis of patient sera with unusual Tf patterns, including those with high amounts of trisialo-Tf and genetic variants [23-27]. CDG serum Tf patterns using the CEofix approach $[11,23,28,29]$, the CAPILLARYS assay $[30,31]$ and laboratory-made assays [32-35] have been reported. Furthermore, CZE was also used to determine CDG Tf patterns in extracts from Guthrie cards onto which the patient serum was applied $[30,36]$. In all these papers, few examples were discussed only with the aim of highlighting the use of CZE for CDG monitoring. The CZE separability of asialo-, monosialo, and disialo-Tf glycoforms present in sera of different types of CDG patients, particularly those found in mixed type I/II CDG patients, has not yet been evaluated. This is an important aspect for the adoption of CZE as screening method for CDG and prompted us to assess it in our laboratory.

In this paper, sera of known type I, type II and mixed type I/II CDG patients (Table I) were analyzed by CZE employing the high-resolution CEofix based assay which is employed for determination of CDT with stringent quality assurance $[17,36]$ and for identification of genetic variants of $\operatorname{Tf}[26,27]$. This assay has a higher resolution for disialo-Tf and trisialo-Tf compared to the CAPILLARYS assay [25] and the CEofix assay configuration promoted by the manufacturer of the reagents [17]. The data were compared to those monitored by GIEF with an assay featuring an immobilized $\mathrm{pH}$ gradient. 


\section{MATERIALS AND METHODS}

\subsection{Chemicals, reagents, origin of samples and preparation of samples}

The chemicals used were of analytical grade if not stated otherwise. Ferric citrate and BSA were purchased from Sigma-Aldrich Chemie (Schnelldorf, Germany). Polyclonal rabbit antihuman Tf antibodies (titer: 2800 mg/L) was from Dako (Glostrup, Denmark). Buffers and reagents of the CEofix CDT kit No. 10-004760 were from Analis (Suarlée, Belgium). The CDT ClinCheck serum control level II was from Recipe (Munich, Germany) and used as control sample. Patient sera were received for the determination of the type of CDG (Table 1) or for the monitoring of CDT. CDG samples of patients 12 and 13 (Table 1) were obtained from the Radboudumc center of expertise for disorders of glycosylation (Nijmegen, The Netherlands). Our own serum was used for investigational work. All samples were stored at -20 or $-70^{\circ} \mathrm{C}$ until use. Patient sera were also analyzed after immunosubtraction according to the procedure reported previously $[17,26]$. Briefly, a $1: 2 \mathrm{v} / \mathrm{v}$ mixture of serum and anti-human Tf antibody solution was incubated for $45 \mathrm{~min}$ at room temperature and centrifuged at $8000 \mathrm{~g}$ and $4^{\circ} \mathrm{C}$ for $20 \mathrm{~min}$. A 2:1 v/v mixture of supernatant aliquot and ferric solution was briefly vortex mixed and analyzed.

\subsection{Instrumentation and running conditions for CZE analysis}

All CZE analyses were performed on a PA800plus CE system (Beckman Coulter) according to Joneli et al. [17] and employed in other work from our laboratory $[25,26,35,37]$. Briefly, a 50 $\mu \mathrm{m}$ id fused-silica capillary (Polymicro Technologies, Phoenix, AZ, USA) of $60.2 \mathrm{~cm}$ (50 cm to the detector) total length was used. For iron saturation, $60 \mu \mathrm{L}$ of serum and $60 \mu \mathrm{L}$ of the 
Fe(III) containing solution of the reagent kit (Analis) were combined in a sample vial and briefly mixed as described before [17]. Alternatively, nanoVials (Sciex, Framingham, MA, USA) were used for which the amount of serum and Fe(III) reagent could be reduced to $5 \mu \mathrm{L}$ each. Samples were injected by vacuum ( $12 \mathrm{~s}$ at $0.5 \mathrm{psi}, 1 \mathrm{psi}=6894.76 \mathrm{~Pa}$ ) and were analyzed at a capillary cartridge temperature of $30.0^{\circ} \mathrm{C}$, an applied voltage of $20.0 \mathrm{kV}$ and having a detection wavelength of $200 \mathrm{~nm}$ (interference filter). Instrument control and peak integration were performed with the 32 Karat software (Beckman Coulter). Peak areas were determined by valley-to-valley integration and the data were evaluated on the basis of corrected peak areas (peak area divided by migration time).

\subsection{Instrumentation and running conditions for isoelectric focusing}

GIEF analyses were made as described previously [38]. Briefly, serum Tf was saturated for 30 min with ferric citrate $(0.4 \mathrm{mM})$ in the presence of sodium hydrogen carbonate $(20 \mathrm{mM})$ and separated on a rehydrated Immobiline DryPlate gel (GE Healthcare Life Sciences, Glattbrugg, Switzerland) with a pH range of 4.0-7.0 using the PhastSystem of Amersham Biosciences (GE Healthcare Life Sciences). Tf was visualized by gel immunoprecipitation using polyclonal rabbit anti-human $\mathrm{Tf}$ antibodies (Dako). The antibody solution was directly applied onto the gel and incubated for $1 \mathrm{~h}$. To remove excess antibodies and non-bound proteins, the gel was washed overnight in $0.9 \% \mathrm{NaCl}$ followed by fixation and staining with Phast-Gel Blue R (GE Healthcare Life Sciences) according to the manufacturer's protocol. 


\section{RESULTS AND DISCUSSION}

High-resolution CZE is routinely employed for the determination of CDT in human serum $[17,37]$ and the monitoring of genetic variants $[26,27]$. The assay comprises mixing of human serum with a Fe(III) ion-containing solution before the analysis of the iron-saturated mixture in a dynamically double coated capillary using a commercial buffer at alkaline pH. For sera with very small amounts of $\mathrm{Tf}$ and in case of interferences with other compounds, Tf has to be immunoextracted before analysis [39]. This high-resolution assay was now used to monitor Tf isoform patterns in sera of CDG patients. Immunosubtraction of Tf and reanalysis of the supernatant revealed the peaks in the electropherograms that are associated with Tf. Furthermore, identification of isoform patterns is accomplished by comparison of migration times with those of a commercial serum control and/or with spiking the unknown sample with a serum of a healthy individual, an alcohol abuser or a patient and reanalysis of the mixture. The amounts of single Tf isoforms are calculated as area $\%$ in relation to the sum of the corrected peak areas of all detected Tf isoforms. The Tf isoform pattern in normal healthy subjects comprises disialo-Tf $(<1.7 \%)$, trisialo-Tf (about 4\%), tetrasialo-Tf isoform (about 80\%), pentasialo-Tf (about 14\%) and hexasialo-Tf (about 1\%). Other isoforms are not detected. Following excessive alcohol ingestion for extended time periods, the amounts of less glycosylated forms of Tf (disialo-Tf $>2 \%$, monosialo-Tf and asialo-Tf) are increasing. Less glycosylated forms of Tf are also found in sera of CDG patients $[23,24,28-34]$, CSF [24] and can be produced in vitro by addition of neuraminidase to serum [16].

This article is protected by copyright. All rights reserved. 
The precision performance in regard to migration times of Tf isoforms and quantification of single Tf isoforms and CDT was evaluated with the ClinCheck level II control serum comprising an asialo-Tf level of about $0.4 \%$ and a disialo-Tf amount of about $3.0 \%$ and regular sample vials comprising $60 \mu \mathrm{L}$ of serum and $60 \mu \mathrm{L}$ of the $\mathrm{Fe}(\mathrm{III})$ solution. RSD values $(n=10)$ for the intraday repeatability of migration times of all Tf peaks were found to be $<$ $0.25 \%$. Corresponding values for the interday data were between 0.33 and $1.09 \%$. RSD values for the quantitative determination of the Tf isoforms were between 1.37 (tetrasialoTf, glycoforms with highest level) and 13.02\% (asialo-Tf, glycoforms with lowest level). The limits of isoform quantification and detection were determined to be 0.10 and $0.05 \%$, respectively [17]. Using nanoVials instead of regular sample vials, for which the amount of serum and Fe(III) reagent solution could be reduced to $5 \mu \mathrm{L}$ each, indistinguishable Tf data were obtained. These data document the excellent precision of the assay.

\subsection{CZE analysis of transferrin isoforms in sera of type-la patients}

The graphs presented in Fig. 1 depict electropherograms obtained with the sera of two patients with phosphomannomutase 2 (PMM2) deficiency which is also referred to as CDG type la. PMM2-CDG is an inherited condition with a defect in the second step of the mannose pathway that affects many parts of the body. The type and severity of problems associated with PMM2-CDG vary widely among affected individuals, sometimes even among members of the same family [3]. As shown previously with the serum of another patient [23], PMM2-CDG Tf patterns are substantially different compared to those monitored with the serum of healthy subjects and alcohol abusers. The patterns are characterized with 
substantial peaks for asialo-Tf, disialo-Tf, tetrasialo-Tf and penta-sialo-Tf (Fig. 1). The magnitude of the tetrasialo-Tf peak is much smaller than that of a normal subject. It can be smaller [23], about equal (Fig. 1A) or larger (Fig. 1B) compared to the disialo-Tf peak. These data compare well with the GIEF results shown as inserts in the panels of Fig. 1.

Furthermore, the migration times for asialo-Tf, disialo-Tf, tetrasialo-Tf and penta-sialo-Tf are the same as those observed with the control sample (upper graphs in panels A and B of Fig. 1). The commercial ClinCheck serum level II used as control sample has a small asialo-Tf peak and an elevated disialo-Tf peak and represents a pattern typically observed with sera of alcohol abusers $[17,37]$. The data suggest that the glycoforms detected in sera of PMM2CDG patients are those observed in alcohol abusers and healthy individuals. This is in agreement with the data of intact Tf molecules obtained by LC-ESI-MS [18-20], HPLC-chipQTOF [21] and CZE-ESI-MS [34]. CZE analyses of mixtures comprising sera of PMM2-CDG patients and alcohol abusers revealed the same result (Fig. 1C). Analysis of a mixture of small aliquots of the PMM2-CDG samples 1 to 5 (Table 1 ) resulted in an electropherogram (graph I of Fig. 1C) similar to those shown in Fig. 1A and 1B. This demonstrates that all corresponding Tf-isoforms in the mixed PMM2-CDG sera comigrate. The data obtained with the serum of an alcohol abuser which exhibits an asialo-Tf peak and a small monosialo-Tf peak is depicted as graph II in Fig. 1C. Analysis of the mixture of the PMM2-CDG sera with that of the alcohol abuser (graph III of Fig. 1C) suggest that asialo-Tf and disialo-Tf glycoforms in these samples are indeed comigrating and could thus be identical. MS 
analyses confirmed their identity and revealed that asialo-Tf lacks both whereas disialo-Tf one of the two carbohydrate chains [18-21,34].

The amount of trisialo-Tf in the pattern of PMM2-CDG patients is much smaller compared to healthy individuals and alcohol abusers. The CZE data presented in Fig. 1 are characterized with an unresolved double peak (Fig. 1A and 1C) or a broad peak (Fig. 1B) for trisialo-Tf. It appears that two different molecules are formed which cannot be distinguished in GIEF (same pl values) but exhibit slightly different effective mobilities under the conditions used for CZE. Such double peaks are typically not observed in patient sera of alcohol abusers as well as in sera of healthy individuals (graph IV of Fig. 1C). The data revealed that trisialo-Tf found in the serum of the alcohol abuser corresponds to the first part of the trisialo-Tf peak monitored in the samples of PMM2-CDG patients (graph III of Fig. 1C). The same was found to be true for trisialo-Tf of a healthy subject (graph IV of Fig. 1C). Complete separation of the two peaks would require an assay of higher resolution. It is interesting to note that large and broad trisialo-Tf peaks that can even overlap the disialo-Tf peak are occasionally observed for sera of patients with liver cirrhosis. This situation is referred to as disialotrisialo-Tf bridging and was reported to be a result of increased branching and fucosylation of the carbohydrate moiety $[24,40]$. Furthermore, sera of PMM2-CDG patients also contain small amounts of monosialo-Tf (Fig. 1). The concentration of this isoform, however, is too small to produce reliable peaks. 


\subsection{Diversity of Tf patterns of type I patients}

The $\alpha-1,3-$ glucosyltransferase (ALG6)-CDG (previously called CDG Ic) is a defect in N-glycan assembly in the endoplasmic reticulum and is, after PMM2-CDG, the second most common type of CDG $[41,42]$. Analysis of the serum of an ALG6-CDG patient revealed a CZE pattern that is essentially identical to that of PMM2 (Fig 2A). The same was found to be true for a serum of a patient with a steroid 5 $\alpha$-reductase type 3 deficiency (Fig. 2B) referred to as SRD5A3-CDG or CDG-Iq [43] and pattern similarity is in agreement with the data obtained by ESI-MS [20].

The data presented in Fig. 3A were obtained with the serum of an ALG13-CDG (CDG-Is) patient. ALG13-CDG is a CDG-I subtype with a near-normal glycosylation such that diagnosis of ALG13-CDG with GIEF can be missed if genetic studies are not performed [44]. The electropherogram shows an asialo-Tf peak ( $0.91 \%$ of total Tf) and an elevated disialo-Tf peak (7.74\%). This Tf profile has a higher level of disialo-Tf compared to trisialo-Tf which is

different than in the control sample used (Fig. 3A). The disialo-Tf levels in the sera of alcohol abusers can be lower, equal or higher than trisialo-Tf $[23,24,17]$. Thus, the ALG13-CDG pattern shown in Fig. 3A cannot be distinguished from that of an alcohol abuser. Migration times of all isoforms are identical to those of the control (upper graph of Fig. 3A). ALG11CDG (CDG Ip) is another CDG-I subtype and as shown in Fig. 3B, a disialo-Tf peak of $6.44 \%$ of total Tf was monitored which is much higher than in a healthy individual. The electropherogram of Fig. 3B does not reveal the presence of an asialo-Tf that could be quantified. This is somewhat different compared to the sera of alcohol abusers for which an 
asialo-Tf peak is typically monitored when the disialo-Tf peak is larger than $3.0 \%[24,45]$. Migration times of the Tf isoforms monitored in panels $3 \mathrm{~A}$ and $3 \mathrm{~B}$ were identical.

\subsection{Transferrin patterns of type II patients}

The data presented in Fig. 4A were obtained with a serum of a MGAT2-CDG (CDG type lla; sample 8 of Table 1) patient. The monitored Tf pattern is completely different compared to those of CDG type I patients. It comprises a peak for disialo-Tf and a broad peak tentatively assigned to trisialo-Tf (Fig. 4A). This pattern is consistent with that obtained by GIEF (insert in Fig. 4A). Comparison with the control sample (upper graph in Fig. 4A) revealed that disialo-Tf in the case of MGAT2-CDG was detected earlier than in the control sample and the PMM2-CDG sera. This was confirmed by analysis of a mixture of the CDG sample and the serum of an alcohol abuser (compare graph III with graphs I and II of Fig. 4C). Disialo-Tf detected in the serum of the MGAT2-CDG patient was thus labeled with an asterisk. It represents a Tf isoform with both carbohydrate chains bearing one terminal sialic acid residue which is in contrast to disialo-Tf that has one carbohydrate chain with two sialic acid residues [18]. The latter is found in sera of CDG type I patients, alcohol abusers and healthy individuals $[18,21]$ whereas the former in CDG type II patients [18,34]. The two disialo-Tf isoforms have the same charge, but differ in mass and thus in electrophoretic mobility. The compound with two carbohydrate chains is detected before that with one chain because it has a larger mass and thus lower electrophoretic mobility. Evidence for this behavior was previously reported by Sanz-Nebot et al. [34]. The monitored Tf pattern is in principle 
comparable to that obtained by low resolution CZE assays [11]. With the latter assay format, however, it is not possible to sense the difference of the two disialo-Tf molecules.

COG5-CDG, also known as CDG type Ili, is an extremely rare form of a CDG syndrome [46]. Analysis of the serum of such a patient (patient 6 of Table 1) revealed the pattern shown in Fig. 4B. The first detected disialo-Tf peak (peak $2^{*}$ ) was found to comigrate with that of the MGAT2-CDG patient sample (compare graphs IV and VI of Fig. 4C). Furthermore, at the time interval of disialo-Tf found in alcohol abusers and healthy individuals, a small peak was monitored (peak 2 in graphs IV to VI of Fig. 4C). Compared to a healthy subject, it revealed a higher amount of disialo-Tf ( $4.26 \%$ instead of $<1.70 \%$ for the sum of peaks 2 and $\left.2^{*}\right)$ and a much higher amount of trisialo-Tf (22.1\% instead of 3-5\% of total Tf). GIEF analysis revealed the presence of disialo-, trisialo-, tetrasialo- and pentasialo-Tf (insert in Fig. 4B).

Furthermore, the GIEF data revealed a pattern with double bands indicating that this person is a Tf-CC heterozygote whose isoforms could not be resolved by CZE (for other examples of such genetic variants see $[26,27])$.

MGAT2- and COG5-CDG belong to the type II CDG group leading to truncated N-glycans.

Disialo-Tf marked with an asterisk (peak $2^{*}$ ) is a result of undersialylated glycans, whereas disialo-Tf (peak 2) deriving from PMM2 patients (type I CDG) represents hypoglycosylated Tf due to an unoccupied N-glycosylation site. It is therefore concluded that high-resolution CZE is able to distinguish between hypoglycosylated and undersialylated disialo-Tf. 


\subsection{Transferrin patterns of mixed type I/II patients}

PGM1-CDG results from deficiencies in phosphoglucomutase 1 and combines glycosylation abnormalities found in CDG type I and CDG type II [47]. This is documented with the CZE data presented in Fig. 5A that shows an electropherogram obtained with the serum of a PGM1-CDG patient (sample 11 of Table 1). It reveals peaks for asialo-Tf, monosialo-Tf, disialo-Tf, trisialo-Tf, tetrasialo-Tf and pentasialo-Tf. The same isoforms were detected by GIEF (insert in Fig. 5A). As for the example of Fig. 4B, the GIEF data show double bands, indicating that it represents a heterozygote Tf-CC variant. The CZE data exhibit two peaks for asialo-Tf, monosialo-Tf and disialo-Tf, but not for the other isoforms (graph I of Fig. 5B). Peaks labeled without asterisk correspond to those detected in alcohol abusers and CDG type I patients (Figs. 1C and 5B). Furthermore, this serum also exhibits peaks for Tf isoforms with undersialylated glycans (peaks marked with asterisk), namely disialo-Tf (peak $2^{*}$ ) that was found in the sera of CDG type II samples shown in Fig. 4, and corresponding peaks for monosialo-Tf and asialo-Tf. These findings are in agreement with the MS analysis of intact Tf molecules reported by Tegtmeyer et al. [47] and Van Scherpenzeel et al. [21]. In the highresolution CZE assay, all three compounds with undersialylated glycans are detected before those with hypoglycosylation. For trisialo-Tf, the PGM1-CDG data (Fig. 5A) and the COG5CDG electropherogram (Fig. 4B) show a normal peak and not a double or broad peak as was observed for PMM2-CDG (Fig. 1). According to the migration time, it corresponds to trisialoTf monitored with the control sample (upper graphs in Figs. 4B and $5 \mathrm{~A}$ ) and the serum of an alcohol abuser (Fig. 4C and 5B).

This article is protected by copyright. All rights reserved. 
A sample from a patient diagnosed with liver cirrhosis, various carcinomas, Parkinson's disease and diabetes mellitus type 2 that was analyzed in the context of CDT monitoring by CZE exhibited unusual desialylated Tf glycoforms [17]. This serum comprised different asialo-Tf, monosialo-Tf and disialo-Tf peaks compared to those of alcohol abusers (Fig. 6A, Fig. 6B with graphs I to III). These peaks were found to comigrate with the three Tf isoforms with undersialylated glycans present in the PGM1-CDG sample of Fig. 5 (Fig. 6B, compare graphs I and IV, peaks marked with asterisks). Thus, it can be hypothesized that this serum is more likely stemming from a patient with a secondary cause of abnormal glycosylation rather than a type I/II CDG disorder. Hyposialylated Tf glycoforms appear to be present in patients with liver diseases. The occurrence, however, is rare as only samples of two patients with such a transferrin pattern were analyzed in a 10-year period during which a total of about 4500 samples from the hepatology outpatient clinic were analyzed. Such hyposialylated Tf glycoforms are also characteristic for sera with neuraminidase activity, including those of patients with hemolytic uremic syndrome associated with a Streptococcus pneumoniae infection $[47,48]$.

\section{CONCLUDING REMARKS}

The high-resolution CZE assay was used to resolve Tf glycoforms of similar structure. Under the described conditions, hypoglycosylated Tf glycoforms of CDG type I and undersialylated Tf glycoforms of CDG type II can be distinguished. For disialo-Tf, monosialo-Tf and asialo-Tf, undersialylated glycoforms are detected ahead of their corresponding hypoglycosylated forms because they have a higher molecular mass and thus a lower electrophoretic mobility.

This article is protected by copyright. All rights reserved. 
The serum of a PGM1-CDG patient is shown to comprise both hypoglycosylated Tf glycoforms found in CDG type I and undersialylated glycoforms of CDG type II. The CZE data indicate that Tf glycoforms monitored in CDG type I samples comigrate with those observed in sera of alcohol abusers. This is in agreement with the MS literature which reports the identity of the glycoforms in these samples [18-21,34]. Most of the monitored Tf profiles of CDG patients are different to those of alcohol abusers or normal subjects and thus provide a mean to recognize the presence of a CDG. The data obtained reveal that the Tf profiles of ALG6-CDG and SRD5A3-CDG patients are the same as those monitored for PMM2-CDG. The ALG13-CDG sample comprises a Tf glycoform distribution that cannot be distinguished from that of an alcohol abuser. The high-resolution CZE assay has the potential to be used as screening method for CDGs. It can be run with a few $\mu \mathrm{L}$ of serum when samples are applied with nanoVials. This is an important feature as only tiny amounts of serum can be drawn from newborns and infants. This aspect was pointed out previously for CDT analysis by CZE in samples of healthy newborns [49]. Furthermore, hyposialylated Tf glycoforms detected in the serum of a patient with liver cirrhosis and other diseases revealed the same electrophoretic behavior as those of the serum of a PGM1-CDG patient.

\section{Acknowledgements}

The authors gratefully acknowledge the excellent work provided by the laboratory technicians who performed the CDT analyses in the routine laboratory and the kind supply of the ALG6-CDG and SRD5A3-CDG samples provided by Dr. Dirk J. Lefeber. 
Conflicts of interest

The authors have declared no conflict of interest.

\section{REFERENCES}

[1] de Jong, G., van Ejik, H.G., Microheterogeneity of human serum transferrin: a biological phenomenon studied by isoelectric focusing in immobilized $\mathrm{pH}$ gradients. Electrophoresis 1988, 9, 589-598.

[2] Arndt, T., Carbohydrate-deficient transferrin as a marker of chronic alcohol abuse: a critical review of preanalysis, analysis, and interpretation. Clin. Chem. 2001, 47, 1327.

[3] Jaeken, J., Congenital disorders of glycosylation. Ann. N.Y. Acad. Sci. 2010, 1214, 190198.

[4] Hennet, T., Diseases of glycosylation beyond classical congenital disorders of glycosylation. Biochim. Biophys. Acta 2012, 1820, 1306-1317.

[5] Freeze, H.H., Chong, J.X., Bamshad, M.J., Ng, B.G., Solving glycosylation disorders: fundamental approaches reveal complicated pathways. Am. J. Hum. Genet. 2014, 94, 161-175.

[6] Péanne, R., de Lonlay, P., Foulquier, F., Kornak, U., Lefeber, D.J., Morava, E., Pérez, B., Seta, N., Thiel, C., Van Schaftingen, E., Matthijs, G., Jaeken, J., Congenital disorders of glycosylation (CDG): Quo vadis? Eur. J. Med. Genet. 2018, in press. doi: 10.1016/j.ejmg.2017.10.012.

This article is protected by copyright. All rights reserved. 
[7] Görg, A., Weser, J., Westermeier, R., Postel, W., Weidinger, S., Patutschnick, W., Cleve, $\mathrm{H}$., Isoelectric focusing with immobilized $\mathrm{pH}$ gradients for the analysis of transferrin (Tf) subtypes and variants. Hum. Genet. 1983, 64, 222-226.

[8] Pascali, V.L., Dobosz, M., Destro-Bisol, G., D’Aloja E., Characterization of genetic variants of human serum transferrin by isoelectric focusing: comparison between conventional and immobilized $\mathrm{pH}$ gradients, and application to a protocol for paternity testing. Electrophoresis 1988, 9, 411-417.

[9] Henry, H., Froehlich, F., Perret, R., Tissot, J.-D., Eilers-Messerli, B., Lavanchy, D., Dionisi-Vici, C., Gonvers, J.-J., Bachmann, C., Microheterogeneity of serum glycoproteins in patients with chronic alcohol abuse compared with carbohydratedeficient glycoprotein syndrome type I. Clin. Chem. 1999, 45, 1408-1413.

[10] Helander, A., Bergström, J., Freeze, H.H., Testing for congenital disorders of glycosylation by HPLC measurement of serum transferrin glycoforms. Clin. Chem. $2004,50,954-958$.

[11] Carchon, H.A., Chevigné, R., Falmagne, J.-B., Jaeken, J., Diagnosis of congenital disorders of glycosylation by capillary zone electrophoresis of serum transferrin. Clin. Chem. 2004, 50, 101-111.

[12] F. Crivellente, G. Fracasso, R. Valentini, G. Manetto, A.P. Riviera, F. Tagliaro, Improved method for carbohydrate-deficient transferrin determination in human serum by capillary zone electrophoresis. J. Chromatogr. B 2000, 739, 81-93. 
[13] Lanz, C., Kuhn, M., Bortolotti, F., Tagliaro, F., Thormann, W., Evaluation and optimization of capillary zone electrophoresis with different dynamic capillary coatings for the determination of carbohydrate-deficient transferrin in human serum. J. Chromatogr. A 2002, 979, 43-57.

[14] Wuyts, B., Delanghe, J.R., Kasvosve, I., Wauters A, Neels H, Janssens J., Determination of carbohydrate-deficient transferrin using capillary zone electrophoresis. Clin. Chem. 2001, 47, 247-255.

[15] Legros, F.J., Nuyens, V., Minet, E., Emonts, P., Zouaoui Boudjeltia, K., Courbe, A., Ruelle, J.-L., Colicis, J., de L’Escaille, F., Henry, J.-P., Carbohydrate-deficient transferrin isoforms measured by capillary zone electrophoresis for detection of alcohol abuse. Clin. Chem. 2002, 48, 2177-2186.

[16] Lanz, C., Marti, U., Thormann, W., Capillary zone electrophoresis with a dynamic double coating for analysis of carbohydrate-deficient transferrin in human serum. Precision performance and pattern recognition. J. Chromatogr. A 2003, 1013, 131147.

[17] Joneli, J., Lanz, C., Thormann, W., Capillary zone electrophoresis determination of carbohydrate-deficient transferrin using the new CEofix reagents under highresolution conditions. J. Chromatogr. A 2006, 1130, 272-280. Hänseler, E., Jaeken, J., Heizmann, C.W., Troxler, H., Mass spectrometric analysis of 
human transferrin in different body fluids. Clin. Chem. Lab. Med. 2003, 41, 15801588.

[19] Lacey, J.M., Bergen, H.R., Magera, M.J., Naylor, S., O'Brien, J.F., Rapid determination of transferrin isoforms by immunoaffinity liquid chromatography and electrospray mass spectrometry. Clin. Chem. 2001, 47, 513-518.

[20] Sturiale, L., Barone, R., Garozzo, D., The impact of mass spectrometry in the diagnosis of congenital disorders of glycosylation. J. Inherit. Metab. Dis. 2011, 34, 891-899.

[21] van Scherpenzeel, M., Steenbergen, G., Morava, E., Wevers, R.A., Lefeber, D.J., Highresolution mass spectrometry glycoprofiling of intact transferrin for diagnosis and subtype identification in the congenital disorders of glycosylation. Transl. Res. 2015, $166,639-649$.

[22] Wada, Y., Mass spectrometry of transferrin glycoforms to detect congenital disorders of glycosylation: Site-specific profiles and pitfalls. Proteomics 2016, 16, 3105-3110.

[23] Caslavska, J., Thormann, W., Monitoring of alcohol markers by capillary electrophoresis. J. Sep. Sci. 2013, 36, 75-95.

[24] Caslavska, J., Thormann, W., Monitoring of transferrin isoforms in biological samples by capillary electrophoresis. J. Sep. Sci. 2018, 41, 303-322.

[25] Marti, U., Joneli, J., Caslavska, J., Thormann, W., Determination of carbohydratedeficient transferrin in human serum by two capillary zone electrophoresis methods 
and a direct immunoassay: Comparison of patient data. J. Sep. Sci. 2008, 31, 30793087.

[26] Caslavska, J., Joneli, J., Wanzenried, U., Schiess, J., Lanz, C., Thormann, W., Determination of Genetic Transferrin Variants in Human Serum by Capillary Zone Electrophoresis. J. Sep. Sci. 2014, 37, 1663-1670.

[27] Caslavska, J., Lanz, C., Burda, P., Tobler, M., Thormann, W., Analysis of genetic variants of transferrin in human serum after desialylation by capillary zone electrophoresis and capillary isoelectric focusing. J. Sep. Sci. 2017, 40, 2488-2497.

[28] Quintana, E., Montero, R., Casado, M., Navarro-Sastre, A., Vilaseca, M.A., Briones, P., Artuch, R., Comparison between high performance liquid chromatography and capillary zone electrophoresis for the diagnosis of congenital disorders of glycosylation. J. Chromatogr. B 2009, 877, 2513-2518.

[29] Casado, M., O’Callaghan, M.M., Montero, R., Pérez-Cerda, C., Pérez, B., Briones, P., Quintana, E., Muchart, J., Aracil, A., Pineda, M., Artuch, R., Mild clinical and biochemical phenotype in two patients with PMM2-CDG (congenital disorder of Glycosylation la). Cerebellum 2012, 11, 557-563.

[30] Parente, F., Ah Mew, N., Jaeken, J., Gilfix, B.M., A new capillary zone electrophoresis method fort the screening of congenital disorders of glycosylation (CDG). Clin. Chim.Acta 2010, 411, 64-66.

This article is protected by copyright. All rights reserved. 
[31] Dave, M.B., Dherai, A.J., Udani, V.P., Hegde, A.U., Desai, N.U., Ashavaid, T.F., Comparison of transferrin isoform analysis by capillary electrophoresis and HPLC for screening congential disorders of glycosylation. J. Clin. Lab. Anal. 2017, e22167, 1-8.

[32] Iourin, O., Mattu, T.S., Mian, N., Keir, G., Winchester, B., Dwek, R.A., Rudd, P.M., The identification of abnormal glycoforms of serum transferrin in carbohydrate deficient glycoprotein syndrome type I by capillary zone electrophoresis. Glycoconj. J. 1996, $13,1031-1042$.

[33] Oda, R.P., Prasad, R., Stout, R.L., Coffin, D., Patton, W.P., Kraft, D.L., O’Brien, J.F., Landers, J.P., Capillary electrophoresis-based separation of transferrin sialoforms in patients with carbohydrate-deficient glycoprotein syndrome. Electrophoresis 1997, $18,1819-1826$.

[34] Sanz-Nebot, V., Balaguer, E., Benavente, F., Neusüss, C., Barbosa, J., Characterization of transferrin glycoforms in human serum by CE-UV and CE-ESI-MS. Electrophoresis 2007, 28, 1949-1957.

[35] Sanz-Nebot, V., González, P., Toro, I., Ribes, A., Barbosa, J., Characterization of human transferrin glycoforms by capillary electrophoresis and electrospray ionization mass spectrometry. J. Chromatogr. B 2003, 798, 1-7.

[36] Carchon, H.A., Nsibu Ndosimao, C., Van Aerschot, S., Jaeken, J., Use of serum on Guthrie cards in screening for congenital disorders of glycosylation, Clin. Chem. $2006,52,774-775$. 
[37] Joneli, J., Wanzenried, U., Schiess, J., Lanz, C., Caslavska, J., Thormann, W., Determination of carbohydrate-deficient transferrin in human serum by capillary zone electrophoresis: Evaluation of assay performance and quality assurance over a 10-year period in the routine arena. Electrophoresis 2013, 34, 1563-1571.

[38] Hülsmeier, A.J., Paesold-Burda, P., Hennet, T., N-Glycosylation Site Occupancy in Serum Glycoproteins Using Multiple Reaction Monitoring Liquid ChromatographyMass Spectrometry. Mol. Cell. Proteomics 2007, 6, 2132-2138, 2007.

[39] Caslavska, J., Joneli, J., Wanzenried, U., Schiess, J., Thormann, W., Transferrin immunoextraction for determination of carbohydrate-deficient transferrin in human serum by capillary zone electrophoresis, J. Sep. Sci. 2012, 35, 3521-3528.

[40] Landberg, E., Åström, E., Kågedal, B., Påhlsson, P., Disialo-trisialo bridging of transferrin is due to increased branching and fucosylation of the carbohydrate moiety. Clin. Chim. Acta 2012, 414, 58-64.

[41] Imbach, T., Burda, P., Kuhnert, P., Wever, R.A., Aebi, M., Berger, E.G., Hennet, T., A mutation in the human ortholog of the Saccharomyces cerevisiae ALG6 gene causes carbohydrate-deficient glycoprotein syndrome type-Ic. Proc. Natl. Acad. Sci. USA $1999,96,6982-6987$.

[42] Morava, E., Tiemes, V., Thiel, C., Seta, N., de Lonlay, P., de Klerk, H., Mulder, M., Rubio-Gozalbo, E., Visser, G., van Hasselt, P., Horovitz, D.D.G., Fischinger Moura de Souza, C., Schwartz, I.V.D., Green, A., Al-Owain, M., Uziel, G., Sigaudy, S., Chabrol, B., 
van Spronsen, F.-J., Steinert, M., Komini, E., Wurm, D., Bevot, A., Ayadi, A., Huijben, K., Dercksen, M., Witters, P., Jaeken, J., Matthijs, G., Lefeber, D.J., Wevers, R.A., ALG6-CDG: a recognizable phenotype with epilepsy, proximal muscle weakness, ataxia and behavioral and limb anomalies. J. Inherit. Metab. Dis. 2016, 39, 713-723.

[43] Wheeler, P.G., Ng, B.G., Sanford, L., Sutton, V.R., Bartholomew, D.W., Pastore, M.T., Bamshad, M.J., Kircher, M., Buckingham, K.J., Nickerson, D.A., Shendure, J., Freeze, H.H., SRD5A3-CDG: Expanding the phenotype of a congenital disorder of glycosylation with emphasis on adult onset features. Am. J. Med. Genet. Part A 2016, $170,3165-3171$.

[44] Galama, W.H., Verhaagen-van den Akker, S.L.J., Lefeber, D.J., Feenstra, I., Verrips, A., ALG13-CDG with Infantile Spasms in a Male Patient Due to a De Novo ALG13 Gene Mutation, JIMD Rep. 2017 Sep 9. doi: 10.1007/8904_2017_53.

Lanz, C., Kuhn, M., Deiss, V., Thormann, W., Improved capillary electrophoresis method for the determination of carbohydrate-deficient transferrin in patient sera. Electrophoresis 2004, 25, 2309-2318.

[46] Paesold-Burda, P., Maag, C., Troxler, H., Foulquier, F., Kleinert, P., Schnabel, S., Baumgartner, M., Hennet, T., Deficiency in COG5 causes a moderate form of congenital disorders of glycosylation. Hum. Mol. Genet. 2009, 18, 4350-4356.

[47] Tegtmeyer, L.C., Rust, S., van Scherpenzeel, M., Ng, B.G., Losfeld, M.-E., Timal, S., Raymond, K., He, P., Ichikawa, M., Veltman, J., Huijben, K., Shin, Y.S., Sharma, V., 
Adamowicz, M., Lammens, M., Reunert, J., Witten, A., Schrapers, E., Matthijs, G., Jaeken, J., Rymen, D., Stojkovic, T., Laforêt, P., Petit, F., Aumaître, O., Czarnowska, E., Piraud, M., Podskarbi, T., Stanley, C.A., Matalon, R., Burda, P., Seyyedi, S., Debus, V., Socha, P., Sykut-Cegielska, J., van Spronsen, F., de Meirleir, L., Vajro, P., DeClue, T., Ficicioglu, C., Wada, Y., Wevers, R.A., Vanderschaeghe, D., Callewaert, N., Fingerhut, R., Schaftingen, E., Freeze, H.H., Morava, E., Lefeber, D.J., Marquardt, T., Multiple Phenotypes in Phosphoglucomutase 1 Deficiency. N. Engl. J. Med. 2014, 370, 533542.

[48] de Loos, F., Huijben, K.M.L.C., van der Kar, N.C.A.J., Monnens, L.A.H., van den Heuvel, L.P.W.J., Groener, J.E.M., de Moor, R.A., Wevers, R.A., Hemolytic Uremic Syndrome Attributable to Streptococcus pneumoniae Infection: A Novel Cause for Secondary Protein N-Glycan Abnormalities, Clin. Chem. 2002, 48, 781-784.

[49] Zamboni, G., Bortolotti, F., Zaffanello, G., de Paoli, G., Tagliaro, F., Carbohydratedeficient transferrin determined in blood microsamples from healthy newborns by using capillary zone electrophoresis. Scan. J. Clin. Lab. Invest. 2007, 67, 191-195.

This article is protected by copyright. All rights reserved. 


\section{Table 1. CDG samples analyzed}

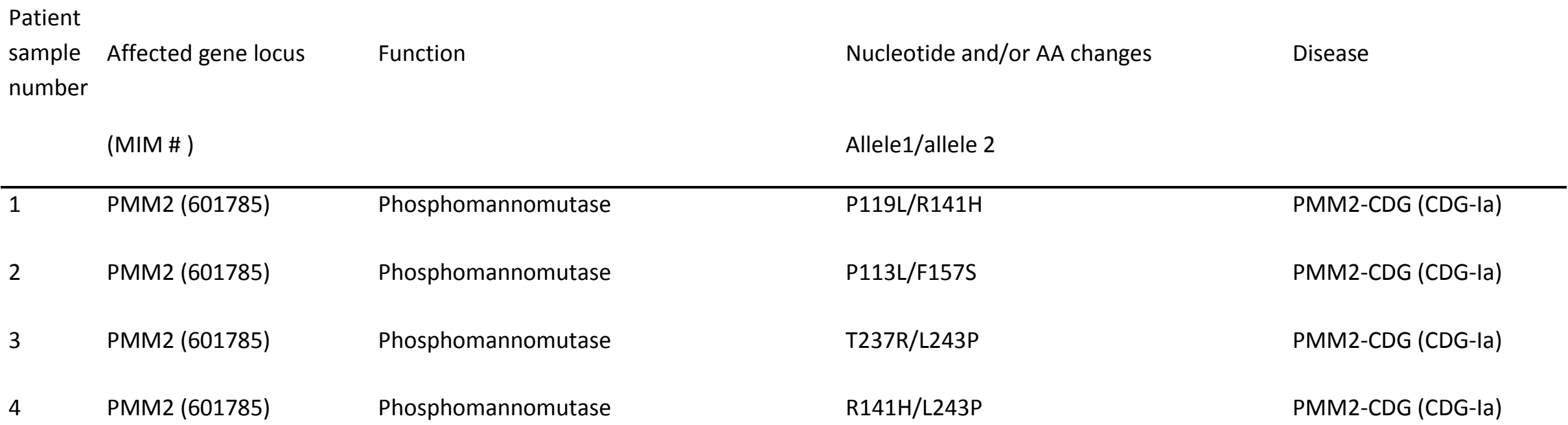

Received: 01 23, 2018; Revised: 04 18, 2018; Accepted: 04 19, 2018

This article has been accepted for publication and undergone full peer review but has not been through the copyediting, typesetting, pagination and proofreading process, which may lead to differences between this version and the Version of Record. Please cite this article as doi: 10.1002/jssc.201800082 .

This article is protected by copyright. All rights reserved. 


\begin{tabular}{|c|c|c|c|c|}
\hline 5 & PMM2 (601785) & Phosphomannomutase & F157S/E197A & PMM2-CDG (CDG-la) \\
\hline 6 & COG5 (606821) & Conserved oligomeric Golgi (COG) complex & c.1669-15T>C/c.1669-15T>C & COG5-CDG (CDG-IIi) \\
\hline 7 & PMM2 (601785) & Phosphomannomutase & c.647A >T (p.N216I)/c.640-9T>G & PMM2-CDG (CDG-la) \\
\hline 8 & MGAT2 (602616) & ß1-2 GlcNAc-transferase & c.1085G>A (W362X)/c.1085G>A (W362X) & MGAT2-CDG (CDG-Ila) \\
\hline 9 & ALG 13 (300884) & $\begin{array}{l}\text { UDP-N-acetylglucosaminyltransferase } \\
\text { subunit }\end{array}$ & mutation not given & ALG13-CDG (CDG-Is) \\
\hline 10 & ALG 11 (613666) & $\alpha 1-2$ man-transferase & mutation not given & ALG11-CDG (CDG-Ip) \\
\hline 11 & PGM1 (614921) & Phosphoglucomutase & mutation not given & PGM1-CDG (CDGI/II) \\
\hline 12 & ALG6 (604566) & a1-3 Glc-transferase & IVS $3+5 \mathrm{~g}>\mathrm{a}$ & ALG6-CDG (CDG-Ic) \\
\hline 13 & SRD5A3 (611715) & Polyprenol reductase & c.29 C>A; p.Ser10X & SRD5A3-CDG (CDG-Iq) \\
\hline
\end{tabular}

This article is protected by copyright. All rights reserved. 


\section{Legends}

Figure 1. CZE electropherograms obtained with the sera of two PMM2-CDG patients. The central graphs are from (A) patient 3 and (B) patient 7 of Table 1. The upper graphs depict data of the commercial control serum with an elevated CDT level and the dashed line graphs were obtained after immunosubtraction of Tf from the patient sera. The inserts depict GIEF data. Panel C presents data of a pool of PMM2-CDG patient sera (samples 1-5 of Table 1, graph I), a serum of an alcohol abuser (graph II), a 1:2 v/v mixture of the PMM2-CDG patient pool and the alcohol abuser (graph III), and the serum of a healthy individual (graph IV). Asialo-Tf, monosialo-Tf, disialo-Tf, trisialo-Tf, tetrasialo-Tf, pentosialo-Tf and hexasialo-Tf are referred to with the numbers $0,1,2,3,4,5$ and 6 , respectively. EO refers to the EOF.
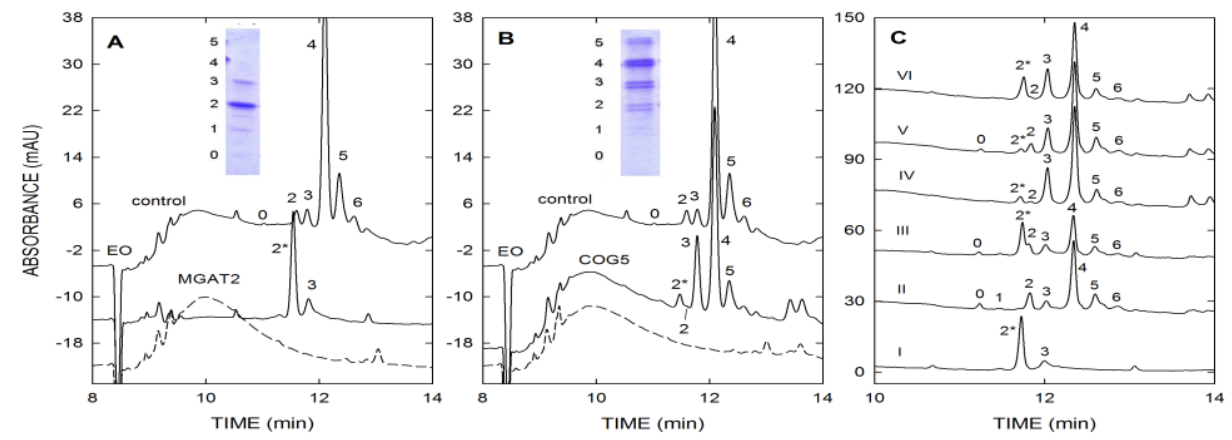

Received: 01 23, 2018; Revised: 04 18, 2018; Accepted: 04 19, 2018

This article has been accepted for publication and undergone full peer review but has not been through the copyediting, typesetting, pagination and proofreading process, which may lead to differences between this version and the Version of Record. Please cite this article as doi: $10.1002 /$ jssc. 201800082 .

This article is protected by copyright. All rights reserved. 
Figure 2. CZE electropherograms obtained with the sera of (A) an ALG6-CDG patient (sample 12 of Table 1) and (B) a SRD5A3-CDG patient (sample 13 of Table 1). Other conditions and key as for Fig. 1.
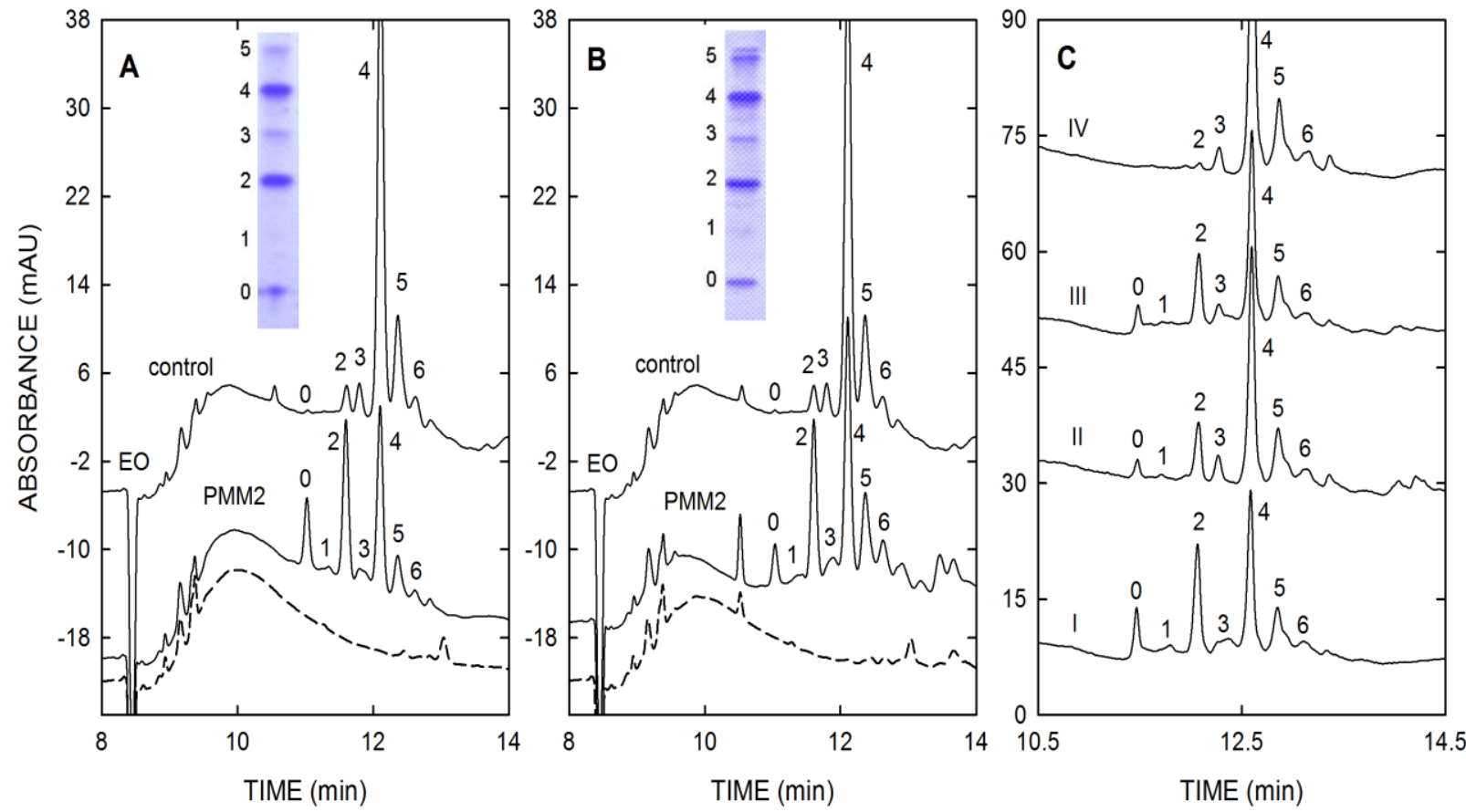

Figure 1

This article is protected by copyright. All rights reserved. 
Figure 3. CZE electropherograms obtained with the sera of (A) an ALG13-CDG patient (sample 9 of Table 1) and (B) an ALG11-CDG patient (sample 10 of Table 1). Other conditions and key as for Fig. 1.
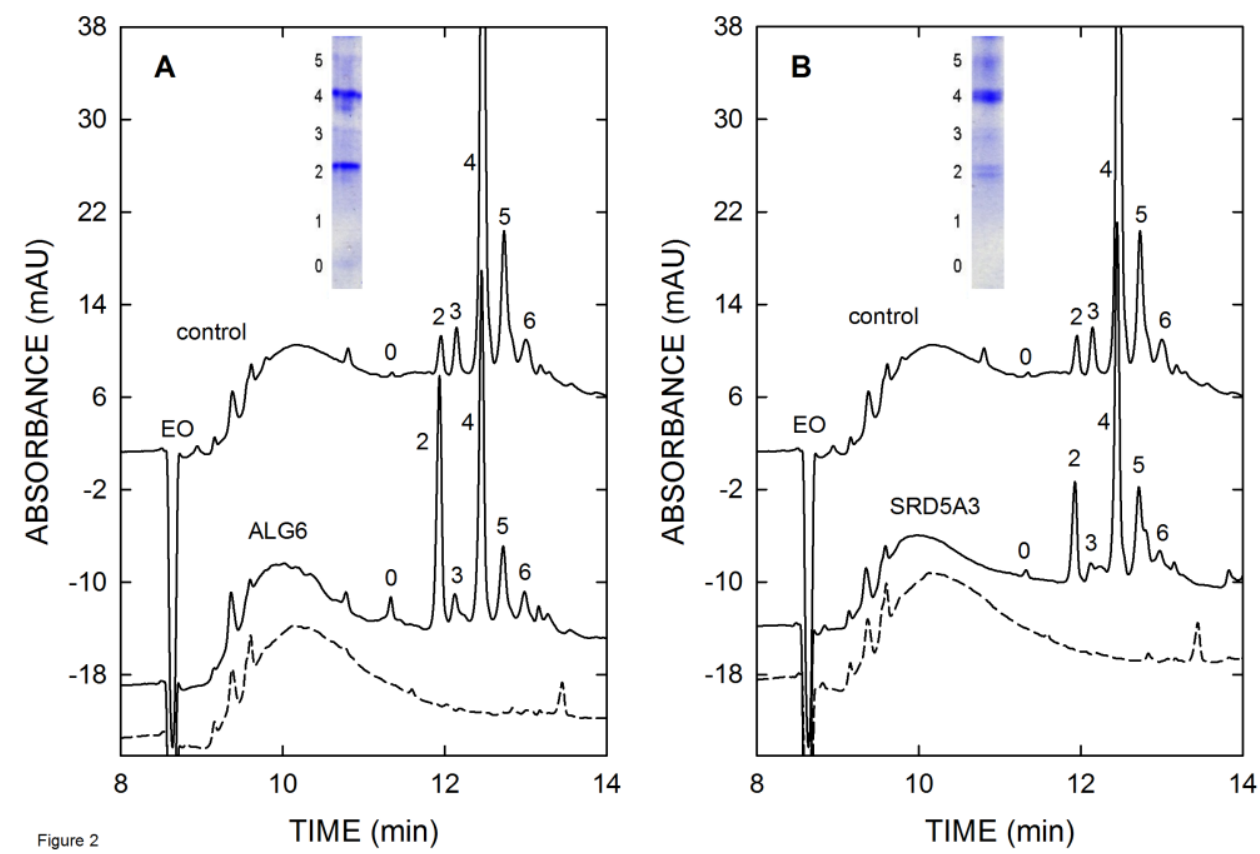

This article is protected by copyright. All rights reserved. 
Figure 4. CZE electropherograms obtained with the sera of (A) a MGAT2-CDG patient (sample 8 of Table 1) and (B) a COG5-CDG patient (sample 6 of Table 1). Panel C depicts data of the MGAT2-CDG serum (graph I), an alcohol abuser (graph II), a 1:1 v/v mixture of the sera of the MGAT2-CDG patient and the alcohol abuser (graph III), the COG5-CDG patient (graph IV), a 1:1 v/v mixture of the sera of the COG5-CDG patient and the alcohol abuser (graph V), and a 2:1 v/v mixture of the sera of the COG5-CDG and MGAT2-CDG patients (graph VI). Other conditions and key as for Fig. 1. The peaks marked with an asterisk are desialylated disialo-Tf of CDG type II with has two carbohydrate moieties featuring one sialic acid each.
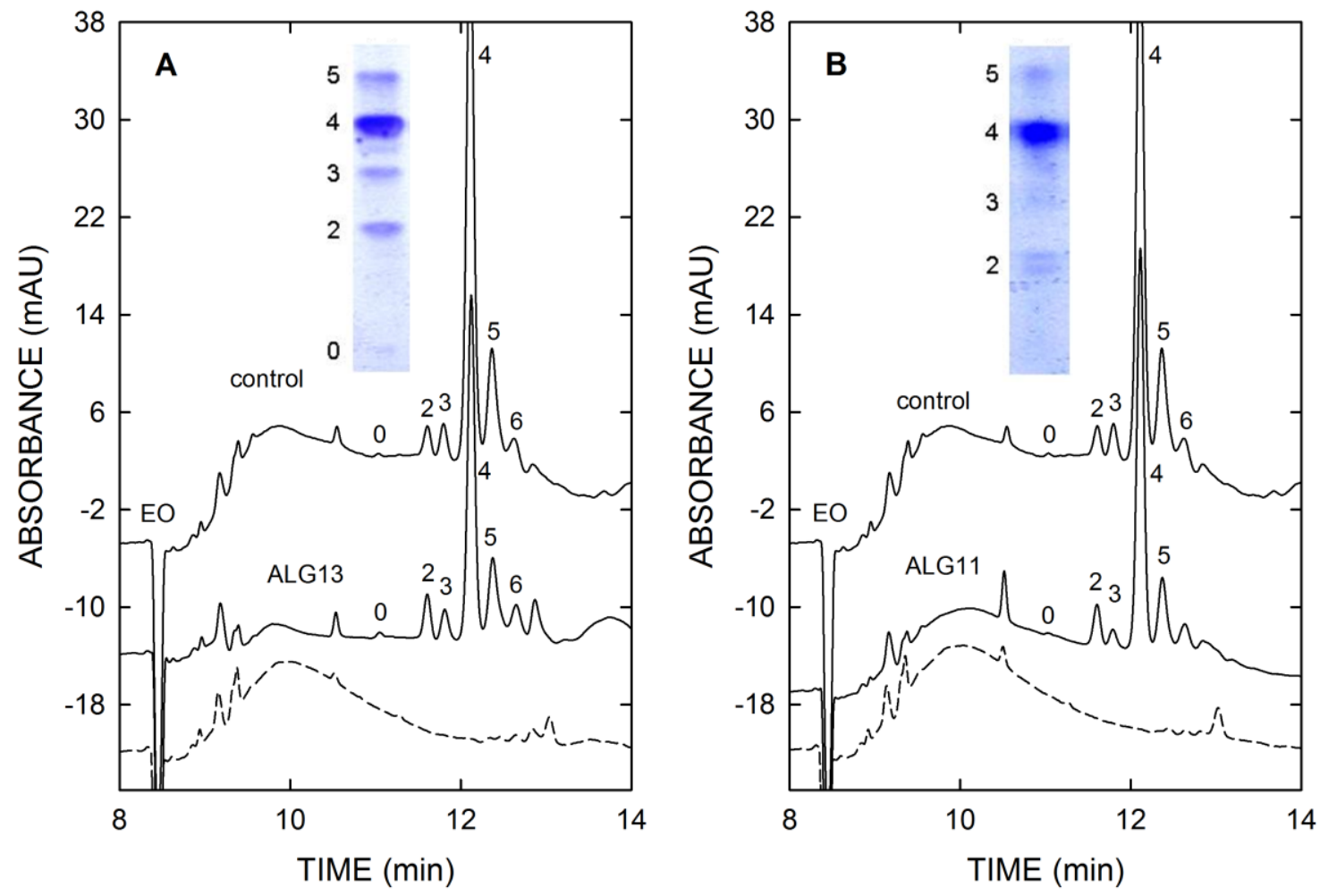

Figure 3

This article is protected by copyright. All rights reserved. 
Figure 5. (A) CZE data obtained with the serum of a PGM1-CDG patient (sample 11 of Table 1) together with the electropherograms obtained after immunosubtraction of Tf in the patient serum (dashed line graph) and the commercial control serum. (B) Data of the PGM1CDG patient sample (graph I), a serum of an alcohol abuser (graph II) and a 1:1 v/v mixture of the sera of the patient and the alcohol abuser (graph III). The peaks marked with asterisks are desialylated Tf glycoforms comprising both carbohydrate chains. Other conditions and key as for Fig. 4.
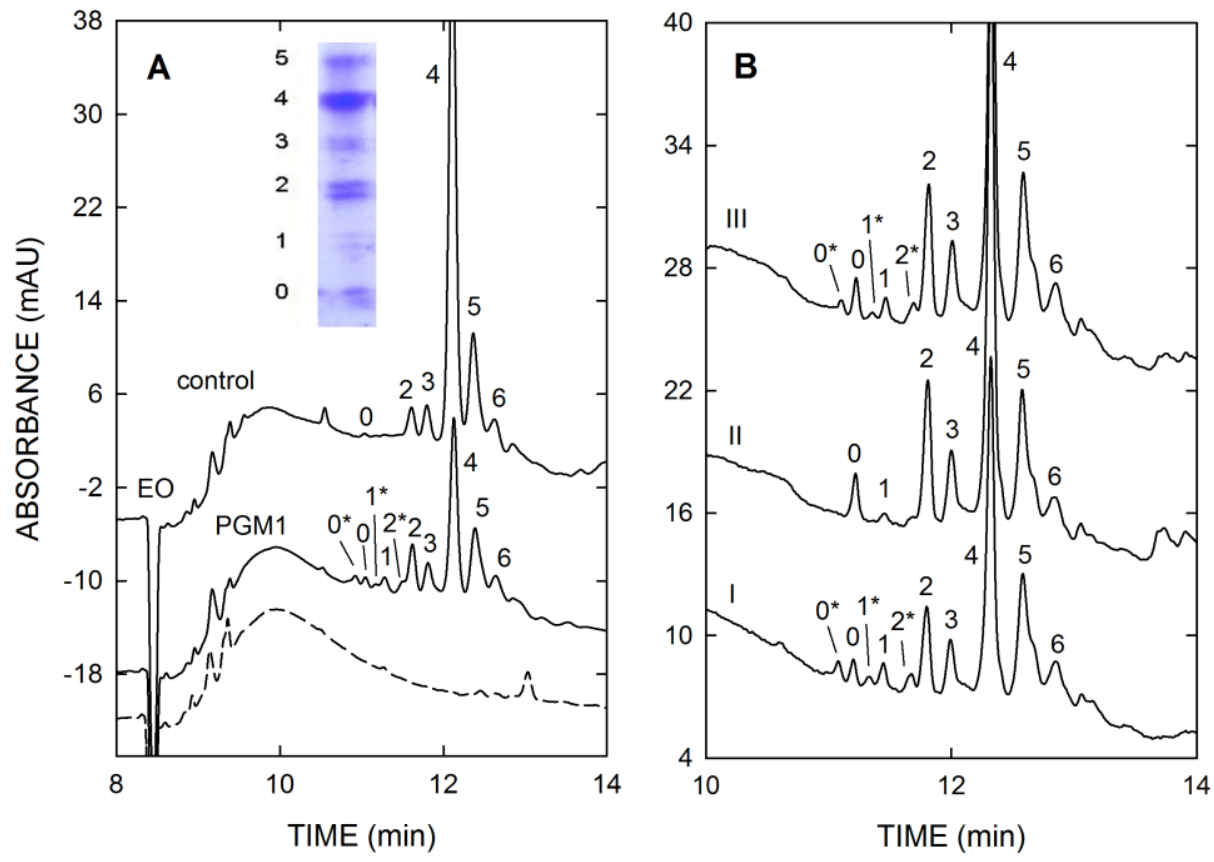

Figure 5

This article is protected by copyright. All rights reserved. 
Figure 6: (A) Data of a patient serum with unusual desialylated Tf isoforms (graph I) together with the electropherogram obtained after immunosubtraction of $\mathrm{Tf}$ in the patient serum (dashed line graph), the serum of an alcohol abuser (graph III) and a 1:1 mixture of the sera of the patient and the alcohol abuser (graph II). (B) Data of the patient serum (graph I), another alcohol abuser (graph II), the 1: $1 \mathrm{v} / \mathrm{v}$ mixture of the patient and the alcohol abuser (graph III) and a 1:2 v/v mixture of the patient serum with that of the PGM1-CDG patient of Fig. 5 (graph IV). Key as for Fig. 5. For further explanations refer to text.
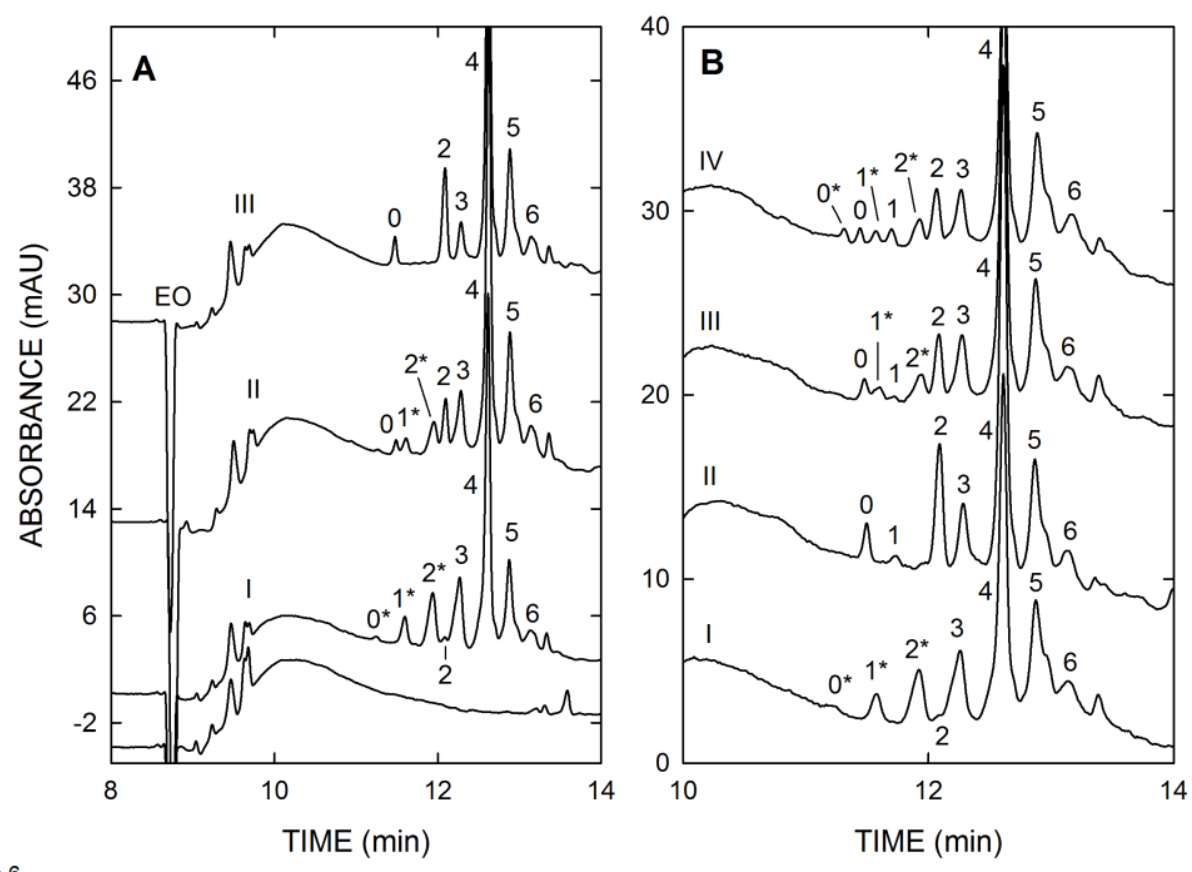

This article is protected by copyright. All rights reserved. 